\title{
Pre-Scheduled Colony Size Variation in Dynamic Environments
}

\author{
Michalis Mavrovouniotis ${ }^{1}$, Anastasia Ioannou ${ }^{2}$, and Shengxiang Yang ${ }^{3}$ \\ 1 School of Science and Technology, Nottingham Trent University \\ Clifton Lane, Nottingham NG11 8NS, United Kingdom \\ michalis.mavrovouniotis@ntu.ac.uk \\ 2 Department of Informatics, University of Leicester \\ University Road, Leicester LE1 7RH, United Kingdom \\ ai630le.ac.uk \\ 3 Centre for Computational Intelligence (CCI) \\ School of Computer Science and Informatics, De Montfort University \\ The Gateway, Leicester LE1 9BH, United Kingdom \\ syang@dmu.ac.uk
}

\begin{abstract}
The performance of the $\mathcal{M} \mathcal{A X}-\mathcal{M I N}$ ant system $(\mathcal{M M A S})$ in dynamic optimization problems (DOPs) is sensitive to the colony size. In particular, a large colony size may waste computational resources whereas a small colony size may restrict the searching capabilities of the algorithm. There is a trade off in the behaviour of the algorithm between the early and later stages of the optimization process. A smaller colony size leads to better performance on shorter runs whereas a larger colony size leads to better performance on longer runs. In this paper, pre-scheduling of varying the colony size of $\mathcal{M M A S}$ is investigated in dynamic environments.
\end{abstract}

\section{Introduction}

Ant colony optimization (ACO) is a metaheuristic inspired by the foraging behaviour of real ant colonies $[2,3]$. ACO algorithms have been successfully applied to many $\mathcal{N} \mathcal{P}$-hard combinatorial problems such as the travelling salesman problem (TSP) [4] and vehicle routing problem (VRP) [7]. In this paper, we focus on a particular ACO variation, i.e., $\mathcal{M A X}-\mathcal{M I N}$ Ant System $(\mathcal{M M A S})[22]$, which is one of the best performing variations.

The construction of solutions from ants is biased by existing pheromone trails and heuristic information. Pheromone trails are updated according to the search experience and towards solution with good quality. This is similar to a learning reinforcement scheme. The behaviour and performance of $\mathcal{M M A S}$ algorithm depends strongly on the number of ants used [5,24]. When a given computational budget is available, e.g., the maximum number of function evaluations, a smaller number of ants will produce more algorithmic iterations whereas a larger number of ants less. Hence, the population size affects the duration of the learning reinforcement [18]. 
In [24], it was investigated that when fewer ants are used, the algorithm may converge quickly at early stages of the optimization process, but get stuck in the stagnation behaviour later on. In contrast, when more ants are used, the algorithm converges slower but to better solutions at later stages of the optimization process. Considering this $\mathcal{M}$ MAS's behaviour, it can be observed that the optimal number of ants depends on the stage of the optimization process. Therefore, $\mathcal{M M A S}$ can benefit from varying the number of ants. For example, a pre-schedule of varying the colony size may improve the performance of $\mathcal{M M A S}$ in dynamic environments. The key idea is to start a few ants when a change occurs and gradually increase the number of ants. In this way, MMAS will benefit from both merits of a small (fast convergence) and large (improve solution quality) colony size at different stages of the optimization process.

Several dynamic test cases of the dynamic TSP (DTSP) are generated for our study. The rest of the paper is organized as follows. Section 2 and Section 3 describe the DOPs generated and the ACO algorithm used for this study, respectively. Section 4 discusses the importance of the population size parameter. Section 5 presents the experimental study with discussions. Finally, Section 6 concludes this paper.

\section{Dynamic Environment}

\subsection{Dynamic Travelling Salesman Problem (DTSP)}

The DTSP is modelled by a fully connected weighted graph $G=(N, A)$, where $N=\left\{v_{1}, \ldots, v_{n}\right\}$ is a set of $n$ nodes (e.g., cities) and $A=\left\{\left(v_{i}, v_{j}\right) \mid v_{i}, v_{j} \in\right.$ $N, i \neq j\}$ is a set of $\operatorname{arcs}$ (i.e., links), where $n$ represents the size of a problem instance. Each arc $\left(v_{i}, v_{j}\right) \in A$ is associated with a non-negative value $d_{i j} \in \mathbb{R}^{+}$, which represents the distance between cities $v_{i}$ and $v_{j}$. The objective of the problem is to find the shortest Hamiltonian cycle that starts from one node and visits each of the other cities once before returning to the starting city.

The distance matrix of the DTSP is subject to changes, which is defined as follows: $\mathbf{D}(t)=\left\{d_{i j}(t)\right\}_{n \times n}$, where $t$ is the period of a dynamic change. A particular TSP solution $s=\left[s_{1}, \ldots, s_{n}\right]$ in the search space is specified by a permutation of the nodes (cities) and it is evaluated as follows:

$$
f(s, t)=d_{s_{n} s_{1}}(t)+\sum_{i=1}^{n-1} d_{s_{i} s_{i+1}}(t) .
$$

\subsection{DTSP Benchmark Generators}

The concept of DTSPs was initially introduced by Psaraftis [20]. Since then, several variations of DTSPs were introduced, where the set of nodes $N[1,9,10$, $12,13,25]$ and/or the cost from the set of arcs $A[6,17,19,21,25]$ cause the weight matrix $\mathbf{W}(t)$ to change during the optimization process. However, there is still no any unified benchmark problem for DTSPs, which makes the comparison with 
algorithms from the literature a very challenging task. One popular benchmark is the DTSP where cities are exchanged: half of the cities from the problem instance are removed to create a spare pool $[9,10,14]$, and the cities from the spare pool are then used to replace cities from the problem instance. Another popular benchmark is the DTSP where the weights of arcs change probabilistically $[25$, 17] (the complete benchmark generator description is given in Section 2.3 since it is the benchmark generator we consider in the experiments). In [6,21], only the weights of arcs that belong to the best tour increase or decrease accordingly.

Younes et al. [26] introduced a benchmark generator for the DTSP with different modes: 1) topology changes as in [10], 2) weight changes in [6], and 3) swap cities. Based on the last mode (i.e., swap cities) of the aforementioned benchmark generator, a general dynamic benchmark generator for permutation-encoded problems (DBGP) was proposed that can generate test cases with known optima [15]. DBGP can convert any stationary TSP instance into a DTSP with specific properties (i.e., frequency and magnitude of changes). Although with DBGP one can observe how close to the optimum an algorithm converges, it sacrifices real-world models for the sake of benchmarking.

\subsection{Generating Dynamic Test Cases}

Considering the problem formulation above, a dynamic test case of a TSP can be generated by modifying the value of the arc between nodes $v_{i}$ and $v_{j}$ as follows:

$$
w_{i j}(T+1)= \begin{cases}w_{i j}(0)+\mathcal{N}(\mu, \sigma), & \text { if }(i, j) \in A_{S}(T) ; \\ w_{i j}(T), & \text { otherwise }\end{cases}
$$

where $T=\lceil t / f\rceil$ is the environmental period index, $f$ is the frequency of change, $t$ is the evaluation count of the algorithm, $\mathcal{N}(\mu, \sigma)$ is a random number generated from a normal distribution with $\mu=0$ and $\sigma=0.2 \times w_{i j}(0), w_{i j}(0)$ is the weight between nodes $v_{i}$ and $v_{j}$ for the initial instance and $A_{S}(T) \subset A$ contains exactly $\lceil m(n(n-1))\rceil$ arcs in which their weights will be subject to changes (either increase or decrease) [25].

Since many real-world problems can be formulated as DTSPs and methods for solving static TSPs can be applied to solve them [8]; the dynamic changes generated in this paper can be generalized and may represent different factors depending on the application. For example, in logistics, the weight changes may represent traffic on the road system or in telecommunications the weight changes may represent delays on the network.

\section{$3 \mathcal{M} \mathcal{A} \mathcal{X}-\mathcal{M} \mathcal{I} \mathcal{N}$ Ant System}

\subsection{Construct Solutions}

One of the state-of-the-art ACO variations is the $\mathcal{M M A S}$ [22]. A colony of $\omega$ ants read pheromones in order to construct their solutions and write pheromones 
to store their solutions. Each ant $k$ uses a probabilistic rule to choose the next node to visit. The decision rule of the $k$ th ant to move from node $v_{i}$ to node $v_{j}$ is defined as follows:

$$
p_{i j}^{k}=\frac{\left[\tau_{i j}\right]^{\alpha}\left[\eta_{i j}\right]^{\beta}}{\sum_{l \in \mathcal{N}_{i}^{k}}\left[\tau_{i l}\right]^{\alpha}\left[\eta_{i l}\right]^{\beta}}, \text { if } j \in \mathcal{N}_{i}^{k},
$$

where $\tau_{i j}$ and $\eta_{i j}$ are the existing pheromone trail and heuristic information available a priori between nodes $v_{i}$ and $v_{j}$, respectively. The heuristic information is defined as $\eta_{i j}=1 / d_{i j}(t)$, where $d_{i j}(t)$ is defined as in Eq. (1). $\mathcal{N}_{i}^{k}$ is the neighbourhood of unvisited nodes incident to node $i$ available for ant $k$ to select. $\alpha$ and $\beta$ are the two parameters which determine the relative influence of $\tau_{i j}$ and $\eta_{i j}$, respectively.

\subsection{Pheromone Update}

The pheromone trails in $\mathcal{M}$ MAS are updated by applying evaporation as follows:

$$
\tau_{i j} \leftarrow(1-\rho) \tau_{i j}, \forall\left(v_{i}, v_{j}\right)
$$

where $\rho$ is the evaporation rate which satisfies $0<\rho \leq 1$, and $\tau_{i j}$ is the existing pheromone value. After evaporation, the best ant deposits pheromone as follows:

$$
\tau_{i j} \leftarrow \tau_{i j}+\Delta \tau_{i j}^{\text {best }}, \forall\left(v_{i}, v_{j}\right) \in T^{b e s t}
$$

where $\Delta \tau_{i j}^{\text {best }}=1 / C^{\text {best }}$ is the amount of pheromone that the best ant deposits and $C^{\text {best }}$ defines the solution quality of tour $T^{\text {best }}$. The best ant that is allowed to deposit pheromone may be either the best-so-far, in which case $C^{b e s t}=C^{b s}$, or the iteration-best, in which case $C^{b e s t}=C^{i b}$, where $C^{b s}$ and $C^{i b}$ are the solution quality of the best-so-far and the iteration best ant, respectively. The best-so-far ant is a special ant that may not necessarily belong to the current population of ants as the iteration best ant. Both update rules are used in an alternate way in the implementation [23].

The lower and upper limits $\tau_{\min }$ and $\tau_{\max }$ of the pheromone trail values are imposed. The $\tau_{\max }$ value is bounded by $1 /\left(\rho C^{b s}\right)$, where $C^{b s}$ is initially the solution quality of an estimated optimal tour and later on is updated whenever a new best-so-far ant solution quality is found. The $\tau_{\min }$ value is set to $\tau_{\min }=$ $\tau_{\max } / 2 n$.

Since only the best ant is allowed to deposit pheromone, the population may quickly converge towards the best solution found in the first iteration. Therefore, the pheromone trails are occasionally reinitialized to the $\tau_{\max }$ value to increase exploration. For example, whenever the stagnation behaviour occurs or when no improved solution is found for a given number of iterations, the pheromone trails are reinitialized. 


\subsection{Adapting to Dynamic Changes}

$\mathcal{M M A S}$ is able to use knowledge from previous environments via pheromone trails and can be applied directly to DOPs without any modifications $[1,16]$. For example, when the changing environments are similar, the pheromone trails of the previous environment may provide knowledge to speed up the optimization process to the new environment. However, the algorithm needs to be flexible enough to accept the knowledge transferred from the pheromone trails, or eliminate the pheromone trails, in order to adapt well to the new environment. In particular, pheromone evaporation enables the algorithm to forget bad decisions made in previous iterations. When a dynamic change occurs, evaporation eliminates the pheromone trails of the previous environment from areas that are generated on the old optimum and helps ants to explore for the new optimum.

In case the changing environments are different, then pheromone reinitialization may be a better choice rather than transferring the knowledge from previous pheromone trails $[1,9,10,16]$. For instance, when a change occurs, the pheromone trails are initialized with an equal amount.

\section{Varying the Colony Size}

\subsection{Effect of the Colony Size in Dynamic Environments}

A previous empirical study showed that the colony size of the $\mathcal{M} \mathcal{M A S}$ algorithm, one of the best performing ACO algorithms, is sensitive to the properties of DOPs [18]. In particular, if for a given DOP only a certain computation budget, e.g., the maximum number of function evaluations, is available, then the colony size, i.e., the number of ants, is a very critical parameter. Since each ant in a colony corresponds to a single function evaluation, an unnecessarily large colony size may waste computations whereas an extremely small colony size may restrict the searching capabilities of ACO.

Furthermore, the colony size has a direct relation with the reinforcement learning period of $\mathrm{ACO}$ because it determines its duration: less ants corresponds to larger duration whereas more ants corresponds to smaller duration. Also the colony size determines how broad the search is at each iteration (e.g., more ants means broader search). Hence, the number of ants needs to be tuned accordingly in order not to waste computation resources and degrade the solution quality.

In this paper, we study the impact of the colony size on the performance of the $\mathcal{M M A S}$ algorithm for DOPs. This kind of problems in a nutshell are a series of stationary optimization problems that all need to be optimized. Therefore, it is straightforward that more challenges exist and the colony size will have impact on the performance of the algorithm. This is because it determines the number of iterations and the broadness of the search as in stationary optimization problems. For example, for a given DOP a predefined computation budged is available between each environmental change that is typically synchronized with the algorithm, i.e., every $f$ evaluations a change occurs [15]. Therefore, an algorithm with a larger colony size means that it will perform a broader search (i.e., 

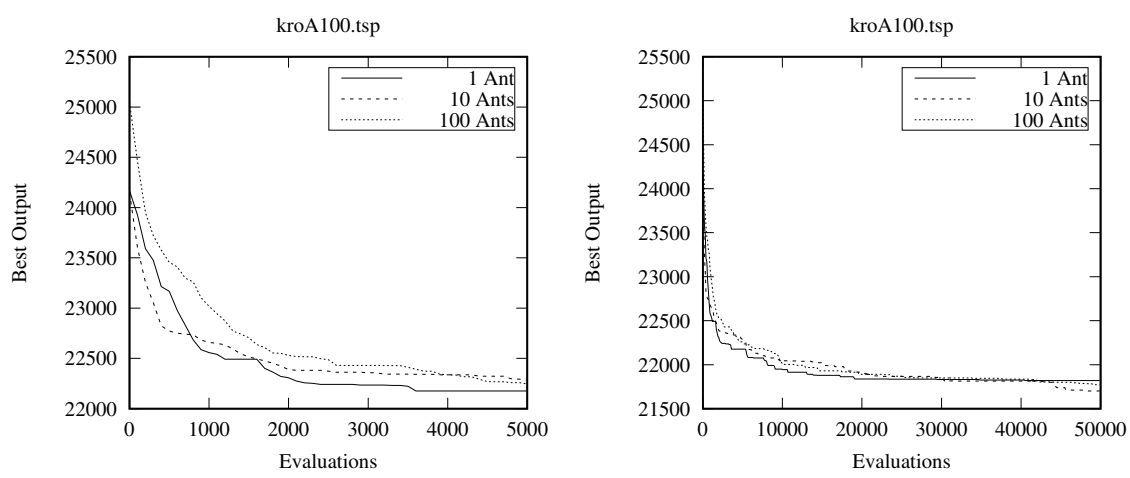

Fig. 1. Performance of $\mathcal{M M A S}$ with different fixed number of ants for a short run of 5000 evaluations (left) and a long run of 50,000 evaluations (right), respectively.

more evaluations per iteration) but it will have limited reinforcement learning (i.e., less number of iteration) for each environmental change.

\subsection{Pre-Scheduling the Colony Size}

The colony size of $\mathcal{M M A S}$ was investigated on the stationary TSP [24]. In particular, the number of ants used shows a trade-off between the early and later optimization process of the algorithm regarding the solution quality. At early stages of the optimization process fewer ants result to better performance, whereas at later stages more ants result to better performance. With fewer ants the algorithm seems to initially progress faster but leads to the stagnation behaviour at later stages. More ants give better results only on later stages of the optimization process. This behaviour of $\mathcal{M} \mathcal{M A S}$ can be observed in Fig. 1. Similar behaviour was observed for other problem instances (kroA150.tsp and kroA200.tsp).

Clearly, at different stages of the optimization process the optimal colony size of $\mathcal{M M A S}$ varies. Therefore, adjusting the colony size during the optimization process seems a better choice rather than keeping a fixed colony size. In fact, pre-scheduling the colony size in stationary environments has proved that it can combine the merits of few ants on shorter runs and the merits of more ants on longer runs [24]. However, in this paper, we are concerned with pre-schedules for dynamic environments. Considering the observations in Fig. 1, a potential good pre-schedule in a dynamic environment could be starting with a small colony size when a change occurs to quickly converge and then gradually increase the colony size to further improve the solution quality.

In particular, four pre-schedules are investigated which are defined as follows:

1. Pre-schedule 1: every 15 iterations add a single ant

2. Pre-schedule 2: every 10 iterations add a single ant

3. Pre-schedule 3 : every 5 iterations add a single ant 
4. Pre-schedule 4: every 2 iterations add a single ant

All schedules start with an initial colony size of 1 ant and increase by 1 ant at a time. When a change occurs the colony size is reset back to 1 and starts to grow until the next dynamic change occurs. An arbitrary number of different preschedules can exist but in this paper we consider these four to determine under which frequency of increasing the number of ants $\mathcal{M} \mathcal{M A S}$ performs better. The size of the colony increases faster from pre-schedule 1 to pre-schedule 2, preschedule 3 and to pre-schedule 4.

\section{Experimental Study}

\subsection{Experimental Setup}

To investigate the effect of the colony size of $\mathcal{M M A S}$ in dynamic environments, three TSP stationary benchmark instances (i.e., kroA100.tsp, kroA150.tsp and kroA200.tsp) were obtained from TSPLIB ${ }^{4}$ and corresponding DOPs are generated using the benchmark generator (described in Section 2.3) with $f$ set to 5000 and 50000 function evaluations, indicating quickly and slowly changing environments, respectively, and $m$ set to $0.1,0.25,0.5$ and 0.75 , indicating slightly, to medium, to severely changing environments, respectively. Totally, a series of 8 dynamic test cases of DTSPs are constructed from each stationary benchmark instance to systematically investigate $\mathcal{M}$ MAS algorithm with the proposed prescheduled colony size against standard fixed colony size.

The colony size of a traditional $\mathcal{M M A S}$ was set to fixed values, i.e., $\omega \in$ $\{1,2,5,10,25,50,100\}$, and the results are compared with the pre-scheduled variation of $\mathcal{M}$ MAS. The remaining parameters were set to typical values for DOPs as follows: $\alpha=1, \beta=5$ and $\rho=0.8$ from our preliminary experiments.

\subsection{Performance Measurement}

For each DTSP, 30 independent runs of the $\mathcal{M}$ MAS were executed. For each run, 25 environments changes were allowed and the best so far ant after a dynamic change was recorded. The overall offline performance [11] is defined as follows:

$$
\bar{P}_{\text {offline }}=\frac{1}{E} \sum_{i=1}^{E}\left(\frac{1}{R} \sum_{j=1}^{R} P_{i j}^{*}\right)
$$

where $E$ is the total number of function evaluations, $R$ is the number of runs, $P_{i j}^{*}$ is the best-so-far after a dynamic change of iteration $i$ of run $j$.

\footnotetext{
$\overline{{ }^{4} \text { http://comopt.ifi.uni-heidelberg.de/software/TSPLIB95/ }}$
} 


\subsection{Results and Discussion}

The offline performance results of the $\mathcal{M M A S}$ algorithm on DTSPs with fixed and pre-scheduled colony sizes are presented in Table 1. Pairwise comparisons between the best fixed colony variation $(1,2,5,10,25,50$ and 100 ants) against the best pre-scheduled colony variation (1, 2, 3 and 4 pre-schedules) using MannWhitney statistical tests are performed. The best variation of one type with a bold value indicates that is significantly better than the best variation of the other type. In case both variations types are in bold it indicates insignificantly difference between them. In Figs. 2 and 3, the dynamic offline performance for quickly and slowly changing environments against the algorithmic evaluations are plotted for the first 10 environments to better understand the behaviour of $\mathcal{M M A S}$. From the experimental results, the following observations can be drawn.
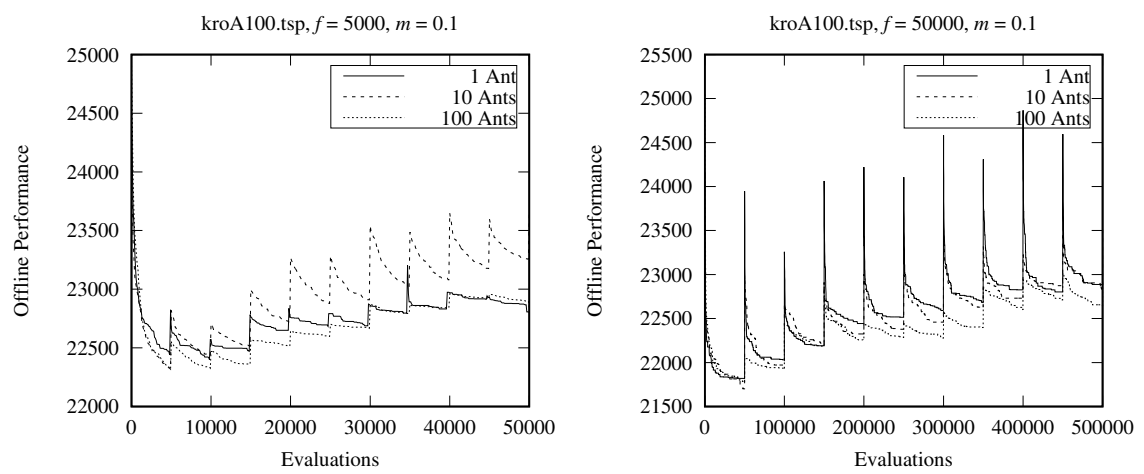

Fig. 2. Dynamic offline performance of $\mathcal{M M A S}$ with different fixed number of ants for quickly changing (left) and slowly changing (right) DTSPs, respectively.

First, the offline performance of fixed colony variations of $\mathcal{M M A S}$ with a larger size is better in most test cases. Only on few cases, i.e., when $m=0.75$, a smaller colony size has better performance. These results were expected for slowly changing DTSPs, i.e., $f=50000$, because more ants perform better in long runs. However, a large colony also performs better for quickly changing DTSPs, i.e., $f=5000$. This is contradictory with the observations in Fig. 1, where a small colony size performed better in a shorter run (corresponds to a quickly changing environment). From Fig. 2, it can be observed that the performance up to the first environment (before any change occurs) the results match the one in Fig. 1. When a dynamic change occurs MMAS with fewer ants perform worst. This is possibly because the pheromone trails generated by fewer ants of the previous environment may not promote exploration when they are used in the new environment. 
Table 1. Comparison of $\mathcal{M} M A S$ variations regarding the results of the offline performance

\begin{tabular}{|c|c|c|c|c|c|c|c|c|}
\hline & \multicolumn{4}{|c|}{$f=5000$} & \multicolumn{4}{|c|}{$\overline{f f=50000}$} \\
\hline$m \Rightarrow$ & 0.1 & 0.25 & 0.5 & 0.75 & 0.1 & 0.25 & 0.5 & 0.75 \\
\hline \# ants & \multicolumn{8}{|c|}{ kroA100.tsp } \\
\hline (1 ant) & 23452 & 23565 & 23417 & 23584 & 22993 & 23125 & 22986 & 23159 \\
\hline (2 ants) & 23378 & 23523 & 23381 & 23538 & 22967 & 23119 & 23004 & 23157 \\
\hline (5 ants) & 23395 & 23575 & 23458 & 23648 & 23014 & 23152 & 23034 & 23188 \\
\hline (10 ant) & 23390 & 23565 & 23410 & 23558 & 22984 & 23151 & 23007 & 23184 \\
\hline (25 ants) & 22831 & 23030 & 23037 & 23085 & 22937 & 23135 & 23002 & 23134 \\
\hline (50 ants) & 22892 & 23072 & 22933 & 23150 & 22880 & 23098 & 22975 & 23109 \\
\hline (100 ants) & 22845 & 23016 & 22964 & 23128 & 22790 & 23092 & 23006 & 23112 \\
\hline Pre-schedule 1 & 23199 & 23385 & 23226 & 23284 & 22826 & 23055 & 22929 & 23055 \\
\hline Pre-sche & 23294 & 23318 & 23126 & 23193 & 22781 & 23038 & 22952 & 23081 \\
\hline Pre-s & 22804 & 23050 & 22740 & 22830 & 22609 & 22913 & 22803 & 22916 \\
\hline Pre-schedule 4 & 22707 & 22887 & 23071 & 23144 & 22814 & 23066 & 22963 & 23090 \\
\hline$\#$ ants & \multicolumn{8}{|c|}{ kroA150.tsp } \\
\hline (1 ant) & 28892 & 29276 & 29473 & 29538 & 28404 & 800 & 397 & 29004 \\
\hline (2 ants) & 28874 & 29260 & 29492 & 29588 & 28436 & 28842 & 29029 & 29060 \\
\hline (5 ants) & 29020 & 29338 & 29610 & 29727 & 28558 & 28907 & 29082 & 29146 \\
\hline (10 ant) & 28993 & 29308 & 29466 & 29568 & 28596 & 28922 & 29088 & 29188 \\
\hline$(25 \mathrm{a}$ & 28600 & 28885 & 29229 & 29419 & 28578 & 28902 & 29057 & 29158 \\
\hline$(50$ & 28575 & 28897 & 29236 & 29300 & 28542 & 28861 & 29056 & 29156 \\
\hline$(100 \mathrm{a}$ & 28596 & 28810 & 29237 & 29302 & 28508 & 28883 & 28966 & 29195 \\
\hline Pre-schedule 1 & 28832 & 29065 & 29163 & 29313 & 28474 & 28870 & 28960 & 29161 \\
\hline Pre-sche & 28856 & 29040 & 29019 & 29309 & 28491 & 28845 & 28995 & 29116 \\
\hline Pre-s & 28566 & 28930 & 29189 & 29286 & 28414 & 28824 & 28994 & 29176 \\
\hline Pre-schedule 4 & 28405 & 28701 & 29334 & 29143 & 28500 & 28843 & 29004 & 29146 \\
\hline$\#$ ants & \multicolumn{8}{|c|}{ kroA200.tsp } \\
\hline (1 ant) & 31799 & 32405 & 32722 & 32829 & 31240 & 31712 & 32044 & 32103 \\
\hline (2 ants) & 31635 & 32178 & 32588 & 32620 & 31219 & 31684 & 32029 & 32061 \\
\hline (5 ants) & 31602 & 32148 & 32561 & 32592 & 31165 & 31656 & 32011 & 32017 \\
\hline (10 ant) & 31543 & 31958 & 32409 & 32327 & 31131 & 31641 & 31992 & 31980 \\
\hline (25 ants) & 31156 & 31529 & 32171 & 32105 & 31077 & 31583 & 31969 & 31987 \\
\hline (50 ants) & 31191 & 31505 & 32138 & 32129 & 31055 & 31552 & 31973 & 31914 \\
\hline (100 ants) & 31158 & 31493 & 32032 & 32113 & 30982 & 31468 & 31915 & 31802 \\
\hline Pre-schedule 1 & 31326 & 31692 & 32134 & 32086 & 31005 & 31503 & 31860 & 31895 \\
\hline Pre-sche & 31243 & 31534 & 31991 & 31850 & 30978 & 31463 & 31841 & 31825 \\
\hline Pre-sch & 31084 & 31466 & 31882 & 32101 & 31002 & 31345 & 31806 & 31718 \\
\hline Pre-schedule 4 & 30935 & 31643 & 31155 & 32248 & 31017 & 31549 & 31861 & 31858 \\
\hline
\end{tabular}

Second, the offline performance of pre-scheduled colony variations of $\mathcal{M M A S}$ that increase the colony size faster, e.g., Pre-schedule 3 and Pre-schedule 4, perform better in most test cases. Only in kroA150.tsp problem instance when $f=50000$ none of the pre-scheduled variations perform better. This is because the results of fixed variation in Table 1 show that 1 ant outperforms other fixed variations. Therefore, a pre-schedule that increases the colony size will not be 

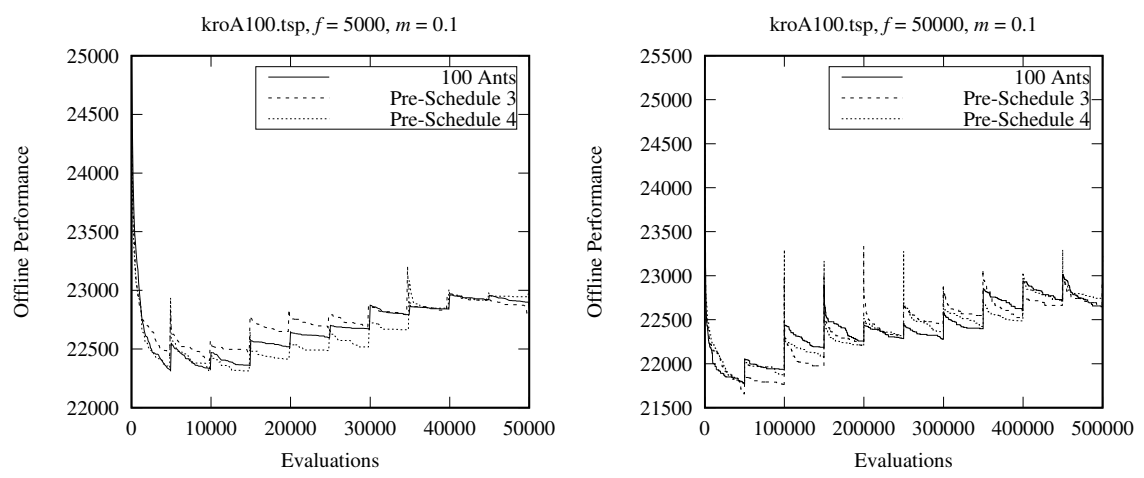

Fig. 3. Dynamic offline performance of the best fixed and pre-schedule $\mathcal{M M A S}$ variations for quickly changing (left) and slowly changing (right) DTSPs, respectively.

helpful. For the remaining cases, either Pre-schedule 3 or Pre-schedule 4 performs better. This shows that the speed of increasing the colony size is problem dependent.

Finally, the comparisons between the fixed and pre-scheduled variation show that with the exception of kroA150.tsp when $f=50000$, the best performing pre-schedule variation outperforms the best performing fixed variation in many DTSPs. From Fig. 3, it can be observed that a pre-scheduled colony size is able to maintain a better offline performance than a fixed colony size.

\section{Conclusions}

The optimal colony size of $\mathcal{M M A S}$ algorithms varies at different stages of the optimization process. More precisely, a small colony size works better for short runs and a large colony size works better for long runs in stationary environments. This paper, investigates different pre-schedules for DTSPs, where $\mathcal{M M A S}$ begins with a single ant and gradually increase its colony size in dynamic environments. When a dynamic change occurs, the colony is reset back to a single ant. The key idea of the pre-schedule is to combine the benefits of small and large colonies. The experiments for different DTSP test cases showed that a varying colony size has a promising performance when compared with a fixed colony size. However, the performance of the pre-schedule of the varying colony strongly depends on the properties of the DTSP. Hence, a direct future work would be to self-adapt the colony size of $\mathcal{M M A S}$. In this way, a possibly automatic pre-schedule will be generated for different DTSPs.

\section{Acknowledgement}

This work was supported by the Engineering and Physical Sciences Research Council (EPSRC) of U.K. under Grant EP/K001310/1. 


\section{References}

1. Angus, D., Hendtlass, T.: Ant colony optimisation applied to a dynamically changing problem. In Hendtlass, T., Ali, M., eds.: Developments in Applied Artificial Intelligence. Volume 2358 of Lecture Notes in Computer Science. Springer Berlin Heidelberg (2002) 618-627

2. Colorni, A., Dorigo, M., Maniezzo, V.: Distributed optimization by ant colonies. In Vaerla, F., Bourgine, P., eds.: Proceedings of the European Conference on Artificial Life, Elsevier Publishing (1991) 134-142

3. Dorigo, M., Maniezzo, V., Colorni, A.: Ant system: optimization by a colony of cooperating agents. IEEE Transactions on Systems, Man, and Cybernetics, Part B: Cybernetics 26(1) (1996) 29-41

4. Dorigo, M., Gambardella, L.M.: Ant colony system: A cooperative learning approach to the traveling salesman problem. IEEE Transactions on Evolutionary Computation 1(1) (1997) 53-66

5. Dorigo, M., Stützle, T.: Ant colony optimization. MIT Press, Cambridge, MA (2004)

6. Eyckelhof, C., Snoek, M.: Ant systems for a dynamic TSP. In Dorigo, M., Di Caro, G., Sampels, M., eds.: Ant Algorithms. Volume 2463 of Lecture Notes in Computer Science. Springer Berlin Heidelberg (2002) 88-99

7. Gambardella, L.M., Taillard, E.D., Agazzi, C.: MACS-VRPTW: A multicolony ant colony system for vehicle routing problems with time windows. In: New Ideas in Optimization. (1999) 63-76

8. Gueta, L., Chiba, R., Ota, J., Arai, T., Ueyama, T.: A practical and integrated method to optimize a manipulator-based inspection system. In: Robotics and Biomimetics, 2007. ROBIO 2007. IEEE International Conference on. (Dec 2007) 1911-1918

9. Guntsch, M., Middendorf, M.: Pheromone modification strategies for ant algorithms applied to dynamic TSP. In Boers, E.J.W., ed.: Applications of Evolutionary Computing. Volume 2037 of Lecture Notes in Computer Science. Springer Berlin Heidelberg (2001) 213-222

10. Guntsch, M., Middendorf, M.: Applying population based ACO to dynamic optimization problems. In Dorigo, M., Di Caro, G., Sampels, M., eds.: Ant Algorithms. Volume 2463 of Lecture Notes in Computer Science. Springer Berlin Heidelberg (2002) 111-122

11. Jin, Y., Branke, J.: Evolutionary optimization in uncertain environments-a survey. IEEE Transactions on Evolutionary Computation 9(3) (2005) 303-317

12. Kang, L., Zhou, A., McKay, B., Li, Y., Kang, Z.: Benchmarking algorithms for dynamic travelling salesman problems. In: Evolutionary Computation, 2004. CEC2004. Congress on. Volume 2. (June 2004) 1286-1292

13. Li, C., Yang, M., Kang, L.: A new approach to solving dynamic tavelling salesman problem. In: 6th Int. Conf. on Simulated Evolution and Learning. (2006) 236-243

14. Mavrovouniotis, M., Yang, S.: A memetic ant colony optimization algorithm for the dynamic travelling salesman problem. Soft Computing 15(7) (2011) 1405-1425

15. Mavrovouniotis, M., Yang, S., Yao, X.: A benchmark generator for dynamic permutation-encoded problems. In Coello, C., Cutello, V., Deb, K., Forrest, S., Nicosia, G., Pavone, M., eds.: Parallel Problem Solving from Nature - PPSN XII. Volume 7492 of Lecture Notes in Computer Science. Springer Berlin Heidelberg (2012) 508-517 
16. Mavrovouniotis, M., Yang, S.: Adapting the pheromone evaporation rate in dynamic routing problems. In Esparcia-Alcázar, A., ed.: Applications of Evolutionary Computation. Volume 7835 of Lecture Notes in Computer Science. Springer Berlin Heidelberg (2013) 606-615

17. Mavrovouniotis, M., Yang, S.: Ant colony optimization with immigrants schemes for the dynamic travelling salesman problem with traffic factors. Applied Soft Computing 13(10) (2013) 4023-4037

18. Mavrovouniotis, M., Yang, S.: Empirical study on the effect of population size on max -min ant sys- tem in dynamic environments. In: Proceedings of the 2016 IEEE Congress on Evolutionary Computation (CEC 2016). (2016) 853860

19. Melo, L., Pereira, F., Costa, E.: Multi-caste ant colony algorithm for the dynamic traveling salesperson problem. In Tomassini, M., Antonioni, A., Daolio, F., Buesser, P., eds.: Adaptive and Natural Computing Algorithms. Volume 7824 of Lecture Notes in Computer Science. Springer Berlin Heidelberg (2013) 179-188

20. Psaraftis, H. In: Dynamic vehicle routing problems. Elsevier (1988) 223-248

21. Simões, A., Costa, E.: Chc-based algorithms for the dynamic traveling salesman problem. In Di Chio, C., Cagnoni, S., Cotta, C., Ebner, M., Ekárt, A., EsparciaAlcázar, A., Merelo, J., Neri, F., Preuss, M., Richter, H., Togelius, J., Yannakakis, G., eds.: Applications of Evolutionary Computation. Volume 6624 of Lecture Notes in Computer Science. Springer Berlin Heidelberg (2011) 354-363

22. Stützle, T., Hoos, H.: $\mathcal{M} \mathcal{A X}-\mathcal{M I N}$ ant system and local search for the traveling salesman problem. In: IEEE International Conference on Evolutionary Computation. (1997) 309-314

23. Stützle, T., Hoos, H.H.: $\mathcal{M} \mathcal{A} \mathcal{X}-\mathcal{M I N}$ ant system. Future Generation Computer Systems 16(8) (2000) $889-914$

24. Stützle, T., López-Ibáñez, M., Pellegrini, P., Maur, M., Montes de Oca, M., Birattari, M., Dorigo, M.: Parameter adaptation in ant colony optimization. In Hamadi, Y., Monfroy, E., Saubion, F., eds.: Autonomous Search. Springer Berlin Heidelberg (2012) 191-215

25. Tinós, R., Whitley, D., Howe, A.: Use of explicit memory in the dynamic traveling salesman problem. In: Proceedings of the 2014 Conference on Genetic and Evolutionary Computation, New York, NY, USA, ACM (2014) 999-1006

26. Younes, A., Calamai, P., Basir, O.: Generalized benchmark generation for dynamic combinatorial problems. In: Proceedings of the 2005 Genetic and Evolutionary Computation Conference, ACM Press (2005) 25-31 